\title{
Potencialidades e fragilidades da política nacional de humanização nos serviços de urgência e emergência sob aperspectiva da enfermagem
}

\section{Potentials and weaknesses of the national humanization policy in urgent and emergency services} from the perspective of nursing

Potencialidades y debilidades de la política nacional de humanización en los servicios de urgencia y emergencia desde la perspectiva de la enfermería

Ana Fátima Souza Melo de Andrade ORCID: https://orcid.org/0000-0002-7024-6175 Centro Universitário Estácio de Sergipe, Brasil E-mail: anafatimamelo@hotmail.com

Weber de Santana Teles ORCID: https://orcid.org/0000-0003-1770-8278 Centro de Hemoterapia de Sergipe, Brasil E-mail: arteecura@hotmail.com

Max Cruz da Silva

ORCID: https://orcid.org/0000-0002-6944-5986 Faculdade Pio Décimo, Brasil E-mail: maxlfi@hotmail.com

Ruth Cristini Torres

ORCID: https://orcid.org/0000-0002-8664-192X Instituto de Hematologia e Hemoterapia de Sergipe, Brasil E-mail: ruthcristini@gmail.com

Ângela Maria Melo Sá Barros ORCID: https://orcid.org/0000-0003-4087-3247 Universidade Federal do Rio de Janeiro, Brasil E-mail: angelsamelo@hotmail.com

Aline Barreto Hora

ORCID: https://orcid.org/0000-0002-3930-6475 Centro Universitário Estácio de Sergipe, Brasil E-mail: aline.barretoh@hotmail.com

Marcel Vinícius Cunha Azevedo ORCID: https://orcid.org/0000-0002-5312-3333

Universidade Tiradentes, Brasil

E-mail: marcelvinicius49@gmail.com

Maria Hozana Santos Silva

ORCID: https://orcid.org/0000-0001-5742-5366 Faculdade Ages de Medicina, Brasil E-mail: hosana_p@hotmail.com Alejandra Debbo

ORCID: https://orcid.org/0000-0002-7743-5921 Universidade Tiradentes, Brasil E-mail: aledebbo@hotmail.com

Taíssa Alice Soledade Calasans ORCID: https://orcid.org/0000-0003-0460-4437 Universidade Tiradentes, Brasil E-mail: taissa.asc@gmail.com

Paulo Celso Curvelo Santos Junior ORCID: https://orcid.org/0000-0001-5834-6782 Universidade Tiradentes, Brasil E-mail: paulo.curvelo.jr@gmail.com

\section{Resumo}

Este estudo tem como objetivo reconhecer as potencialidades e fragilidades relacionadas ao atendimento em urgência e emergência que interferem diretamente na humanização da assistência de enfermagem. Trata-se de uma revisão integrativa, cujo recorte temporal foi de 2015 a 2020, sendo utilizadas as bases de dados PubMed, BVS, LILACS, BDENF, CINAHL e SciELO. As principais fragilidades foram: falta de capacitação profissional, sobrecarga de trabalho, superlotação do serviço e déficit de recursos humanos, falta de equipamentos, má gestão dos leitos 
credenciados e conflitos interprofissionais. Em relação às potencialidades, observou-se que a escuta ativa e o acolhimento com classificação de risco realizado pelo enfermeiro são fatores que contribuem, de forma positiva, para implementação da PNH. Portanto, conclui-se que o enfermeiro juntamente com sua equipe busca constantemente superar novos desafios, sua autonomia está estreitamente relacionada aos saberes próprios da profissão, com a finalidade de executar funções com poder e qualidade, fundamentados no saber científico, reconhecido como o principal instrumento de trabalho do enfermeiro. Neste sentido, a classificação de risco emerge como uma importante ferramenta do cuidado em enfermagem, posto que o enfermeiro gerencia a superlotação, pois,é notório que muitos usuários, em função de não receberem um atendimento satisfatório em unidades básicas de saúde ou na Atenção Especializada, acabam superlotando os serviços de urgência e emergência.

Palavras-chave: Fragilidades; Potencialidades; Enfermagem; Serviços de urgência e emergência.

\begin{abstract}
This study aims to recognize the strengths and weaknesses related to urgent and emergency care that directly interfere in the humanization of nursing care. This is an integrative review, whose time frame was from 2015 to 2020 , using the databases PubMed, BVS, LILACS, BDENF, CINAHL and SciELO. The main weaknesses were: lack of professional training, work overload, service overcrowding and lack of human resources, lack of equipment, poor management of accredited beds and interprofessional conflicts. Regarding the potential, it was observed that active listening and welcoming with risk classification performed by the nurse are factors that contribute positively to the implementation of the PNH. Therefore, it is concluded that nurses and their team constantly seek to overcome new challenges, their autonomy is closely related to the knowledge of the profession, in order to perform functions with power and quality, based on scientific knowledge, recognized as the main instrument of the nurse's work. In this sense, risk classification emerges as an important tool in nursing care, since the nurse manages overcrowding, as it is notorious that many users, due to not receiving satisfactory care in basic health units or in Specialized Care, end up overcrowding urgent and emergency services.
\end{abstract}

Keywords: Weaknesses; Potentials; Nursing; Urgent and emergency services.

\title{
Resumen
}

Este estudio tiene como objetivo reconocer las fortalezas y debilidades relacionadas con la atención de urgencia y emergencia que interfieren directamente en la humanización del cuidado de enfermería. Se trata de una revisión integradora, cuyo marco temporal fue de 2015 a 2020, utilizando las bases de datos PubMed, BVS, LILACS, BDENF, CINAHL y SciELO. Las principales debilidades fueron: falta de formación profesional, sobrecarga de trabajo, masificación de servicios y falta de recursos humanos, falta de equipamiento, mala gestión de las camas acreditadas y conflictos interprofesionales. En cuanto al potencial, se observó que la escucha activa y la acogida con clasificación de riesgo realizada por la enfermera son factores que contribuyen positivamente a la implementación de la PNH. Por lo tanto, se concluye que el enfermero y su equipo buscan constantemente la superación de nuevos desafíos, su autonomía está estrechamente relacionada con el conocimiento de la profesión, con el fin de desempeñar funciones con poder y calidad, con base en el conocimiento científico, reconocido como el principal instrumento de la profesión. el trabajo de la enfermera. En este sentido, la clasificación de riesgos surge como una herramienta importante en el cuidado de enfermería, ya que la enfermera maneja el hacinamiento, ya que es notorio que muchos usuarios, por no recibir una atención satisfactoria en las unidades básicas de salud o en la Atención Especializada, terminan hacinamiento de urgencia y emergencia servicios.

Palabras clave: Debilidades; Potenciales; Enfermería; Servicios de urgencia y emergencia.

\section{Introdução}

De acordo com a Resolução nº 617, de 23 de agosto de 2019, publicada no diário oficial da união, a saúde é uma concessão de todas as pessoas e incumbência do Estado, é considerado um triunfopara todos que vivem no Brasil. A partir de 1988 com o aparececimento do Sistema Único de Saúde (SUS), surge a proposta da universalidade, a integralidade e a eqüidade, no atendimento a saude, aseverando não apenas a inexitência da enfermidade, mas um sistema de qualidade de vida (Brasil, 2021)

Também chamada de Humaniza SUS, a Política Nacional de Humanização (PNH) foi instituída em 2003, pelo Ministério da Saúde, e se refere a uma política pública transversal que abrange todo o processo de trabalho em saúde de forma integral, cuja o procedimento operacional ocorre atévés de metodologia e mecanismos, baseados no tergêmino de incorporação de indivíduos, emblemáticos na saúde como usuários, gestores e trabalhadores (Almeida et al., 2019).

A Política Nacional de Humanização (PNH) é traçada a partir de princípios e diretrizes, cuja operacionalização se dá por meio de métodos e dispositivos pautados na tríplice inclusãode sujeitos representativos na saúde:usuários, gestores e 
trabalhadores (Santos, 2009 \& Brasil, 2004). Almejando mudanças e transformações na atenção e gestão dos serviços de saúde brasileiro. No SUS, os métodos que compõem o sistema de região e hierarquia segundo o graus de complexidade no que diz respeito a atenção nas diversas etapas, anelam modificações na meneira do planejamento a aperacionalização das ações (Brasil, 2014).

A humanização se destaca como política de saúde, tendo como premissa a gestão compartilhada como método e dispositivo na produção de novos modos de gerir e produzir saúde. Diante disso, o usuário suscita uma assistência segura com garantia de conforto e bem-estar nos serviços de saúde, em que ele possa participar efetivamente na discussão das políticas públicas de saúde, em todas as esferas de governo (Ferreira et al., 2021).

Os serviços de urgência e emergência são caracterizados por alguns desafios no que se refere à implementação efetiva da PNH, haja vista que exige o emprego de tecnologia dura e empenho dos profissionais de enfermagem na assistência à clientela, que por vezes, tem sua qualidade reduzida em função do dimensionamento inadequado do quadro de colaboradores e de outras especificidades relacionadas ao ambiente e natureza do trabalho (Assis et al., 2016).

O processo de trabalho nos serviços de urgência e emergência é dinâmico e heterogêneo, envolve ações que podem interferir na saúde dotrabalhador, provocando, dessa forma, maior vulnerabilidade aos riscos ocupacionais, oque expõe tanto o profissional quanto a assistência prestada. Condições de trabalho insalubres, sobrecarga de serviços e carência de recursos são algumas fragilidades encontradas nesses serviços (Silva, 2018).

Perante o exposto, convém ressaltar que os profissionais de enfermagem que laboram nesses serviços precisam desenvolver competências para lidar com demandas diversas e imprevisíveis. Sendo assim, os conhecimentos, habilidades, competências, o raciocínio clínico e a tomada de decisão são elementos indispensáveis à prestação de uma assistência individualizada e humanizada. Portanto, o acolhimento com a escuta ativa, a comunicação, o diálogo e a resolutividade são premissas básicas nesseprocesso (Bellucci et al., 2015).

A liderança do enfermeiro deve concentrar, entre outras características, na identificação dos sinais e sintomas e no estado clínico atual do paciente. Esse tipo de atendimento requer cuidados muito específicos para identificar quais as prioridades, a fim de prevenircomplicações e garantir uma assistência adequada, visando obter o resultado esperadopor toda a equipe de enfermagem (Amestoy, 2016).

Incorporado na PNH há um serviço que é o Acolhimento com Classificação de Risco, o qual prioriza o atendimento conforme a gravidade de cada situação específica. A Classificação de Risco é um serviço que facilita a humanização do atendimento de toda a equipe hospitalar, selecionando a partir de critérios específicos os pacientes que tem maior necessidade no atendimento (Mendes, 2018).

A PNH também denominada Humaniza-SUS visa humanizar o atendimento, tendo como princípios doutrinários: a universalidade, integralidade e equidade. Estes agregam legitimidade ao Sistema Único de Saúde (SUS) e propõe como objetivo geral aplicar a promoção de saúde no cotidiano do atendimento (Andrade et al., 2017).

Esta política visa reorganizar os processos de trabalho em saúde, à proporção que busca transformar as relações sociais, a partir da discussão entre trabalhadores, gestores e usuários. Sob essa ótica, a PNH fundamenta-se em um conjunto de princípios e diretrizes que garantem a efetivação da humanização nas práticas de saúde em qualquer estabelecimento assistencial desaúde (Brasil, 2004).

Apartir de dezembro de 2010, através da Portaria n 4.279, o Ministério da Saúde já iniciava o amadurecimento da proposta para as Redes de Atenção à Saúde (RAS), asegurando fundamentos de consolidação do SUS, buscando promover a universalidade e integralidade da atenção, a equidade. Entretanto, este relato através da portaria, demonstra conceitos fundamentais, necessários afim de que as organização das RAS, nos territórios, tencionando transpor, a divisão do atendimento e da gestão nas regiões de saúde, aperfeiçoando o desempenho político-institucional do SUS (Brasil, 2013). 
Nesta perspectiva, a Rede de Atenção às Urgências e Emergências (RUE) reúne as seguintes diretrizes: Universalidade, equidade e integralidade no cuidado em todas as situações de urgência e emergência; formação de relações horizontais, ampliação do acesso; classificação de risco; regionalização da saúde e atenção territorial; humanização da atenção; organização na forma de trabalhar através de equipes multidisciplinares; práticas clinicas cuidadoras; centralidade nas necessidades de saúde da população; qualificação da atenção e da gestão, entre outras (Brasil, 2011).

A RUE, na sua complexidade considera as desiguais conjunturas, sendo constituida por etapas diferentes de atendimento, afim de atender as condutas e intervenções, em situações de urgência. Sendo assim ha necessidade que seus membros atuem de maneira integral, articulada e simutanea. Desta forma todos os envolvidos devem seguir um programa de qualificação profissional que possa abrager diversos aspectos desde o acolhimento, informação, regulação e treinamento, como demonstrado na Figura 1 (Brasil, 2013).

Figura 1 - Componentes da Rede de Atenção às Urgências e Emergências (RUE) e suas interfaces.

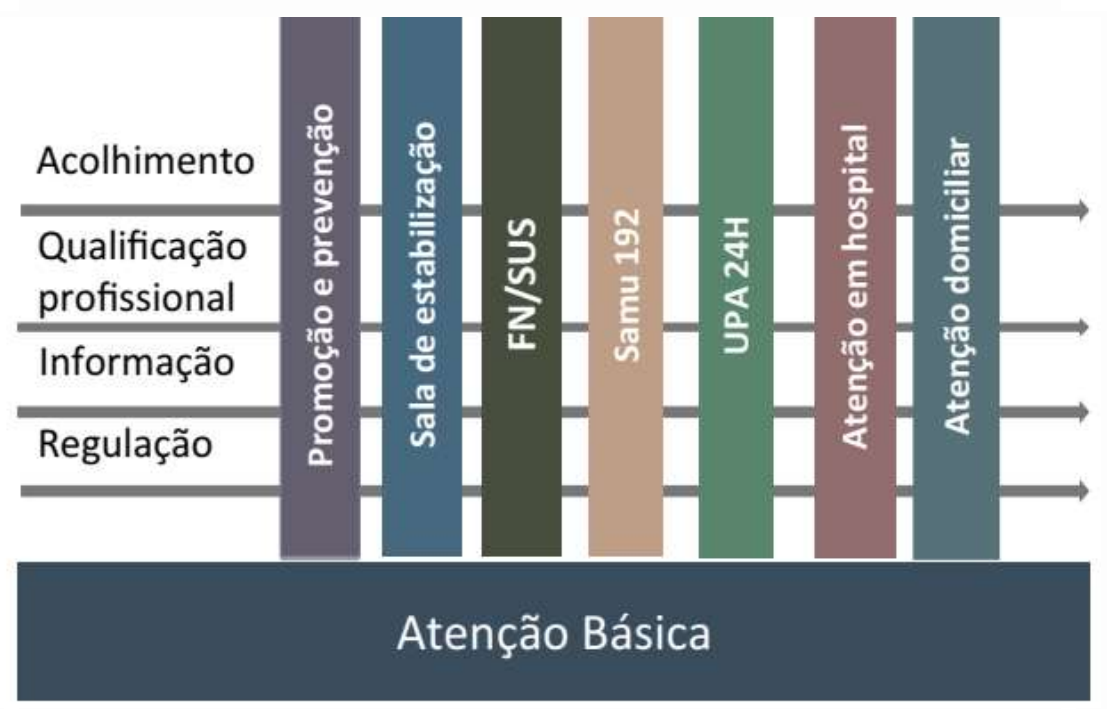

Fonte: SAS/MS (2011).

O enfermeiro exerce um papel primordial na humanização, principalmente, no que tange ao acolhimento, visto que ele é responsávelpor realizar a primeira consulta com o paciente, tornando-se aberto para ouvir asqueixas do usuário através da escuta ativa qualificada. Também incumbe a este profissional realizar a classificação de risco de forma efetiva e resolutiva, o que contribuisignificativamente para a humanização em urgência e emergência (Souza, 2017).

No que concerne ao desenvolvimento de uma visão de cuidado mais humanizada nos setores de urgência e emergência seria necessário distribuir melhor entre os profissionais de saúde os serviços, de forma que a atenção fosse direcionada a um cuidado mais humano,buscando a dignidade das pessoas e fortalecendo o estado psicológico nesse momento que merece uma atenção especial (Gerônimo, Monteles \& Girão, 2020).

O setor de emergência é uma área hospitalar voltada ao acolhimento de pacientes em situações críticas, tais como: ferimentos graves, traumatismos, acidentes com animais peçonhentos, acidente vascular cerebral, entre outros. Por essa razão, os profissionais de enfermagem devem estar capacitados para lidar com situações altamente estressantes, tomando atitudes e decisões para queo estresse não interfira na qualidade desse atendimento (Moraes, 2016).

A necessidade de formação técnica é vista como um aspecto essencial no contexto dos serviços de urgência e emergência, visto que o profissional precisa agregar valores à sua prática clínica, a fim de que possa atender todas as demandas 
inerentes ao serviço. Diante ao exposto, vale destacar que os conhecimentos e as habilidades específicas precisam ser aperfeiçoadas em prol de um atendimento mais individualizado, digno e humanizado (Silva \& Vriesmann, 2019)

Dentre os serviços na área de saúde, os serviços de urgência e emergência são essenciais para garantir assistência à população e são constantemente requisitados com frequência. São serviços que fazem parte do SUS, contudo em virtude da demanda significativa de atendimentos acaba por sobrecarregar o sistema, que já não tem uma boa estrutura física e funcional. Diante dessas circunstâncias, estes serviços acabam sendo comprometidos em relação à qualidade da oferta de serviços (Pereira,Ruzzon \& Martins, 2018).

Para conduzir esta revisão integrativa, foi elaborada a seguinte pergunta de pesquisa: Quais as potencialidades e fragilidades relacionadas ao atendimento em urgência e emergência que interferem diretamente na humanização da assistência de enfermagem?

Deste modo, este estudo tem como objetivo: reconhecer as potencialidades e fragilidades relacionadas ao atendimento em urgência e emergência que interferem diretamente na humanização da assistência de enfermagem.

\section{Metodologia}

Trata-se de uma revisão integrativa da literatura, de caráter exploratório e descritivo com enfoque quantitativo. Este tipo de estudo possibilita sumarizar pesquisas consolidadas e obter conclusões acerca de uma temática. Exige padrões de rigor, clareza e replicação (Moura et al., 2015). Este tipo de revisão está estruturada em seis etapas, a saber: identificação do tema, questão de pesquisa; critérios para inclusão e exclusão de estudos/amostragem ou busca na literatura; definição das informações a serem retiradas dos estudos selecionados/categorização dos estudos; avaliação dos estudos incluídos; interpretação dos resultados; e apresentação da revisão/síntese do conhecimento.

Os dados foram extraídos a partir da síntese da literatura, cujo recorte temporal será de 2015 a 2020 na National Library of Medicine (PubMed) e Biblioteca Virtual em Saúde (BVS), indexadas nas base de dados da Medical Literature Analysis (MedLine), Literatura Latino-Americana (LILACS), Base de Dados da Enfermagem (BDENF), Cumulative Index to Nursing and Allied Health Literature (CINAHL) e Scientific Electronic Library Online (SciELO), foram utilizados os Descritores em Ciências da Saúde (DeCS): urgência; enfermagem; humanização da assistência; acolhimento; classificação de risco, sendo que o cruzamento dos descritores foi realizado através dosoperadores booleanos OR e AND.

Foram utilizados os seguintes critérios de inclusão: artigos publicados na íntegra nas bases de dados supracitadas entre os anos de 2015 a 2020, disponíveisnos idiomas: Português, Inglês e Espanhol. Os critérios de exclusão contemplaram: resumos repetidos em bases de dados diferentes, Teses, dissertações, estudos de revisão, reportagens, editoriais, resumos em anais, além dos estudos que não atendam à questão norteadora.

A análise dos estudos obedeceu a seguinte sequência: leitura dos títulos e leitura dinâmica dos resumos, e por fim, leitura na íntegra, sendo escolhidos posteriormente aqueles artigos que atenderam aos critérios de inclusão e exclusão.Para fins de análise, utilizou-se a análise de conteúdo de Bardin, que se subdivide em quatro etapas: leitura flutuante, que é o estabelecimento de contato com os documentosda coleta de dados, momento em que se começa a conhecer o texto; escolha dos documentos, que consiste na demarcação do que será analisado; formulação dashipóteses e dos objetivos; referenciação dos índices e elaboração de indicadores, que envolve a determinação de indicadores por meio de recortes de texto nos documentosde análise (Bardin, 2010).

\section{Resultados e Discussão}

Após a rigorosa analise de dados, os artigos escolhidos para compor o presente estudo foram determinados a partir dos critérios de inclusão e exclusão, como demonstrado na Figura 1. 
Figura 1. Fluxograma na modalidade prisma dos artigos rastreados nas bases de dados PubMed, BVS,LILACS, BDENF, CINAHL e SciELO, no período de 2015 a 2020.

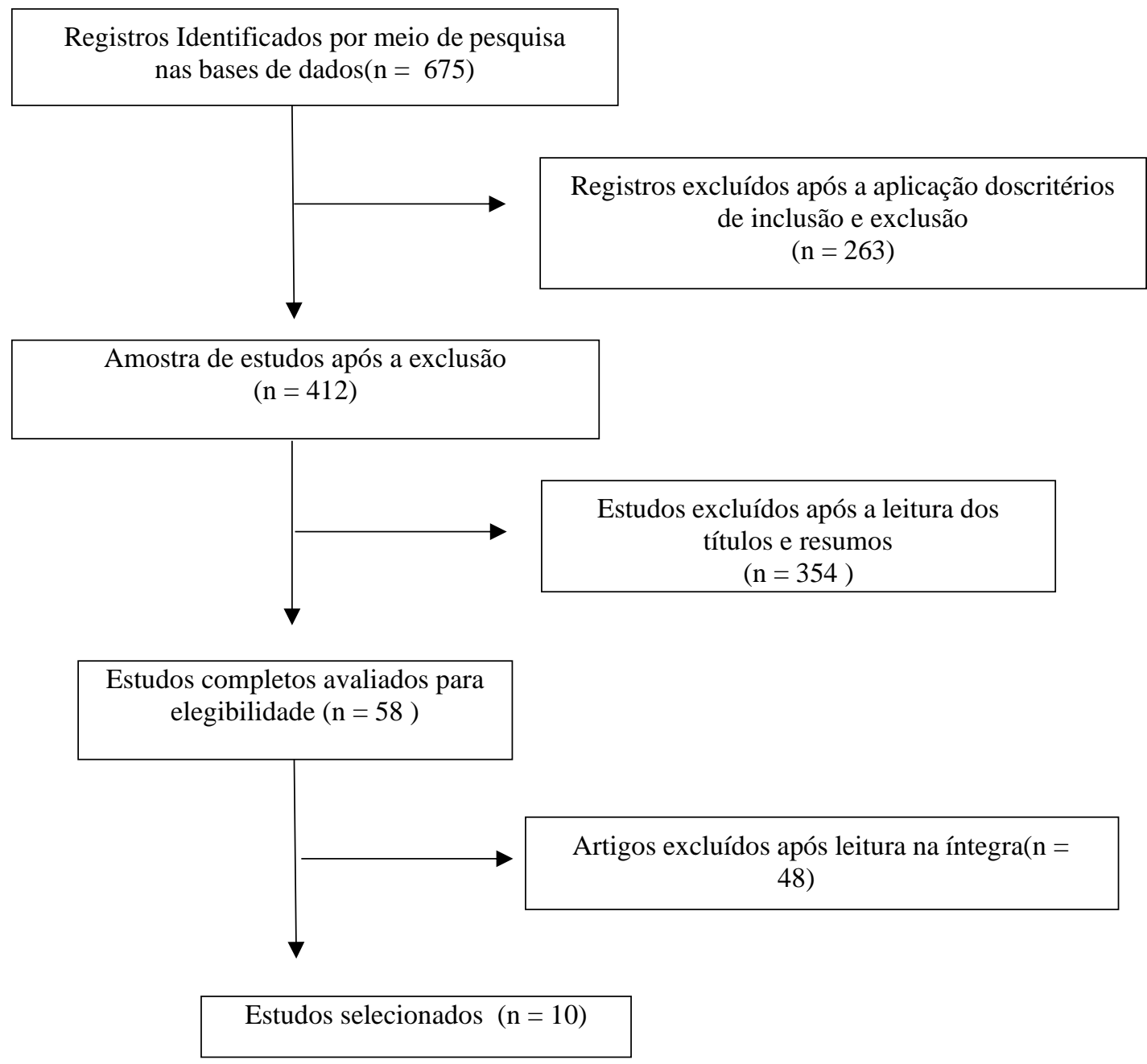

Fonte: Autores (2021).

Embora as políticas de saúde tenham evoluído ao longo do tempo, a implementação das diretrizes da PNH e, notadamente, o acolhimento ao usuário em todos os níveis do sistema, ainda persiste na cultura populara concepção da procura por soluções em serviços de pronto atendimento ou de emergência hospitalar. Com base nisso, a dinâmica de funcionamento dos serviços de urgência e emergência evidencia que o usuário, muitas vezes, não é atendido com presteza, de forma humanizada e acolhedora, devido as razões já expostas (Soares, Brasileiro, \& Souza, 2018).

Seguindo a análise pormenorizada, os artigos foram agrupados no Quadro 1, apresentando, a posteriori, as principais contribuições dos autores sobre a temática em questão. 
Quadro 1. Apresentação dos estudos selecionados para constituir a revisão integrativa.Aracaju, Sergipe. 2021.

\begin{tabular}{|c|c|c|c|c|}
\hline $\begin{array}{c}\text { AUTOR E } \\
\text { ANO }\end{array}$ & REVISTA & TÍTULO & OBJETIVO & $\begin{array}{l}\text { DESENHO DO } \\
\text { ESTUDO }\end{array}$ \\
\hline $\begin{array}{r}\text { Rodrigues et al., } \\
\text { (2019). }\end{array}$ & $\begin{array}{l}\text { Saúde em } \\
\text { Revista }\end{array}$ & $\begin{array}{l}\text { Acolhimento com } \\
\text { classificação de risco } \\
\text { em unidades de pronto } \\
\text { atendimento: avaliação } \\
\text { sob a ótica do } \\
\text { enfermeiro }\end{array}$ & $\begin{array}{c}\text { Avaliar a percepção dos } \\
\text { enfermeiros frente ao } \\
\text { Acolhimento com } \\
\text { Classificação de Risco } \\
\text { implantado emUnidades de } \\
\text { Pronto Atendimento (UPA). }\end{array}$ & $\begin{array}{l}\text { Estudo } \\
\text { descritivo de } \\
\text { caráter corte } \\
\text { transversal. }\end{array}$ \\
\hline $\begin{array}{r}\text { Moraes,Neto \& } \\
\text { Santos. (2020) }\end{array}$ & $\begin{array}{l}\text { Global } \\
\text { Academic } \\
\text { Nursing } \\
\text { Journal }\end{array}$ & $\begin{array}{l}\text { A classificação de } \\
\text { risco em urgência e } \\
\text { emergência: os } \\
\text { desafios da } \\
\text { enfermagem }\end{array}$ & $\begin{array}{l}\text { Conhecer a percepção do } \\
\text { profissional enfermeiro frente à } \\
\text { classificação de risco em um } \\
\text { Hospital de Referência em } \\
\text { Urgência e Emergência em } \\
\text { Traumatologia e Ortopedia de } \\
\text { Santa Catarina. }\end{array}$ & $\begin{array}{l}\text { Pesquisa } \\
\text { qualitativa com } \\
\text { abordagem } \\
\text { exploratória e } \\
\text { descritiva. }\end{array}$ \\
\hline $\begin{array}{r}\text { Roncalli et al., } \\
\text { (2017) }\end{array}$ & $\begin{array}{l}\text { Revista Baiana } \\
\text { de Enfermage } \\
\text { m }\end{array}$ & $\begin{array}{c}\text { Protocolo de } \\
\text { Manchester e } \\
\text { população usuáriana } \\
\text { classificação derisco: } \\
\text { visão do enfermeiro }\end{array}$ & $\begin{array}{l}\text { Compreender a visão do } \\
\text { enfermeiro sobre a utilização } \\
\text { do protocolo de Manchester e } \\
\text { a população usuária na } \\
\text { classificação de risco de uma } \\
\text { Unidade de Pronto } \\
\text { Atendimento. }\end{array}$ & $\begin{array}{l}\text { Estudo de caso } \\
\text { qualitativo } \\
\text { fundamentado na } \\
\text { Sociologia } \\
\text { Compreensiva do } \\
\text { Cotidiano. }\end{array}$ \\
\hline $\begin{array}{r}\text { Leite et al., } \\
\text { (2018) }\end{array}$ & $\begin{array}{l}\text { Ciência, } \\
\text { Cuidado e } \\
\text { Saúde }\end{array}$ & $\begin{array}{c}\text { Os desafios da } \\
\text { humanização dentrode } \\
\text { unidades de pronto } \\
\text { atendimento:a visão } \\
\text { dos gestores }\end{array}$ & $\begin{array}{l}\text { Identificar os desafios da } \\
\text { humanização dentro de } \\
\text { Unidades de Pronto } \\
\text { Atendimento na visão dos } \\
\text { gestores. }\end{array}$ & $\begin{array}{l}\text { Estudo exploratório } \\
\text { comabordagem } \\
\text { qualitativa. }\end{array}$ \\
\hline $\begin{array}{l}\text { Oliveira et } \\
\text { al., (2018). }\end{array}$ & $\begin{array}{l}\text { Revista de } \\
\text { Enfermagem } \\
\text { da UFPI }\end{array}$ & $\begin{array}{l}\text { Avaliação do grau de } \\
\text { superlotaçãa de serviço } \\
\text { hospitalar deurgência }\end{array}$ & $\begin{array}{c}\text { Avaliar o grau de superlotação } \\
\text { do serviço hospitalar de } \\
\text { urgência do Piauí. }\end{array}$ & $\begin{array}{l}\text { Pesquisa } \\
\text { descritiva, } \\
\text { prospectiva, } \\
\text { transversal. }\end{array}$ \\
\hline Silva. (2020) & $\begin{array}{c}\text { Enfermagem } \\
\text { Brasil }\end{array}$ & $\begin{array}{l}\text { Superlotação dos } \\
\text { serviços de urgênciae } \\
\text { emergência hospitalar }\end{array}$ & $\begin{array}{l}\text { Descrever a concepção dos } \\
\text { profissionais de saúde frente à } \\
\text { superlotação dos serviços de } \\
\text { urgência e emergência } \\
\text { hospitalar. }\end{array}$ & $\begin{array}{l}\text { Estudo transversal, } \\
\text { descritivo, com } \\
\text { abordagem } \\
\text { quantitativa. }\end{array}$ \\
\hline $\begin{array}{r}\text { Sokolski, } \\
\text { Vandresen,\& } \\
\text { Senff. (2019) }\end{array}$ & $\begin{array}{l}\text { Saúde e meio } \\
\text { ambiente: } \\
\text { Revista } \\
\text { interdisciplinar }\end{array}$ & $\begin{array}{c}\text { Desafios da } \\
\text { enfermagem para } \\
\text { atuação em urgênciae } \\
\text { emergência }\end{array}$ & $\begin{array}{c}\text { Identificar os desafios } \\
\text { profissionais para a atuação } \\
\text { na urgência e emergência na } \\
\text { área hospitalar. }\end{array}$ & $\begin{array}{l}\text { Estudo } \\
\text { exploratório, } \\
\text { quali- } \\
\text { quantitativo. }\end{array}$ \\
\hline $\begin{array}{r}\text { Pereiraet al., } \\
(2020)\end{array}$ & $\begin{array}{l}\text { Research, } \\
\text { Society and } \\
\text { Development }\end{array}$ & $\begin{array}{l}\text { Atuação do } \\
\text { enfermeiro no serviço } \\
\text { de atendimento pré- } \\
\text { hospitalar: } \\
\text { potencialidades, } \\
\text { fragilidades e } \\
\text { perspectivas }\end{array}$ & $\begin{array}{l}\text { Identificar as potencialidades e } \\
\text { fragilidades vivenciadas pelo } \\
\text { enfermeiro no cotidiano de } \\
\text { trabalho do serviço de } \\
\text { atendimento pré-hospitalar. }\end{array}$ & $\begin{array}{l}\text { Pesquisa } \\
\text { exploratório- } \\
\text { descritiva, } \\
\text { qualitativa. }\end{array}$ \\
\hline $\begin{array}{r}\text { Pereira et } \\
\text { al., }(2020)\end{array}$ & $\begin{array}{l}\text { Revista } \\
\text { Brasileira de } \\
\text { Enfermagem }\end{array}$ & $\begin{array}{ll}\text { Fragilidades } & \text { e } \\
\text { potencialidades } & \\
\text { laborais: percepção de } \\
\text { enfermeiros } & \text { do } \\
\text { serviço móvel de } & \text { de } \\
\text { urgência } & \end{array}$ & $\begin{array}{l}\text { Conhecer a percepção dos } \\
\text { enfermeiros acerca do seu } \\
\text { processo de trabalho em um } \\
\text { Serviço de Atendimento Móvel } \\
\text { de Urgência. }\end{array}$ & $\begin{array}{c}\text { Estudo } \\
\text { qualitativo }\end{array}$ \\
\hline $\begin{array}{r}\text { Soares, } \\
\text { Brasileiro,\& } \\
\text { Souza. (2018) }\end{array}$ & $\begin{array}{l}\text { Revista } \\
\text { Recien- } \\
\text { Revista } \\
\text { Científica de } \\
\text { Enfermagem }\end{array}$ & $\begin{array}{l}\text { Acolhimento com } \\
\text { classificação de risco: } \\
\text { atuação doenfermeiro } \\
\text { na urgência e } \\
\text { emergência }\end{array}$ & $\begin{array}{l}\text { Analisar a assistência de } \\
\text { enfermagem aos pacientes } \\
\text { atendidos com classificaçãode } \\
\text { risco. }\end{array}$ & $\begin{array}{c}\text { Estudo } \\
\text { qualitativo. }\end{array}$ \\
\hline
\end{tabular}


Um estudo conduzido por Rodrigues et al., (2019) teve como amostra 35 profissionais, os quais foram questionados sobre o acolhimento no setor declassificação de risco, dimensões de Estrutura, Processo e Resultados. A dimensão Processo obteve a classificação precária, podendo ser atribuída à falta de conhecimento e domínio sobre a PNH, bem como, a escassez de capacitações da equipe que atuam nos serviços de urgência e emergência. As dificuldades no atendimento aos usuários promovem insegurança em ambas as partes, prejudicam a melhoria da qualidade do assistencial, e se opõem a PNH, no que concerne ao fluxo de atendimento aos usuários e ao conhecimento das condutas do acolhimento com classificação de risco.

Todavia, Moraes, Neto, \& Santos, (2020), citam que a adoção do protocolo de classificação de risco pela instituição de saúde possibilita resultados audaciosos, tais como: redução da taxa de mortalidade; diminuição da fila de espera dos pacientes na recepção; priorização do atendimento aos pacientes mais graves. Além disso, cabe ressaltar que, antes da implementação do protocolo supracitado, os usuários eram atendidos por ordem de chegada, desconsiderando-se a equidade, o que desencadeava maior número de agravos.

A superlotação inviabilzia a oferta de um atendimento integral, o que produz uma assistência fragmentada, com um foco direcionado às ações imediatas e específicas diante da queixa apresentada. A precaridade das instalações físicas, a procura por atendimento de usuários em condições cllínicas não urgentes, os confilitos entre médicos e enfermeiro, e a desarticulação da Rede de Atenção às Urgências com a Atenção Primária, constituem fragilidades para implementar a classificação de risco (Roncalli et al., 2017).

Autores como Leite et al. (2018) e Oliveira et al., (2018) são categóricos em afirmar que o número de leitos credenciados não consegue contemplar todos os pacientes admitidos e internados, além do que o tempo para ser atendido perpassa o tempo estimado para a atuação da equipe. Atrelado a isso, não há monitores e ventiladores para atender a demanda diária de clientes, visto que esses equipamentos são vinculados ao leito credenciado.

Nesta perspectiva, a pesquisa de Silva, (2020) demonstra que a superlotação foi o principal gargalo que justifica a sobrecarga de trabalho e isso claramente dificulta o planejamento da assistência e, consequentemente, a implementação das ações serviços específicos. Observou-se que $97 \%$ dos entrevistados responderam que a superlotação provoca sobrecarga de trabalho. Isso sedeve a maior procura dos usuários para resolutividade de agravos clínicos e traumáticos, o que suscita recursos humanos capacitados para atender essa clientela específica.

Para Sokolski, Vandresen e Senff, (2019) os principais gargalos estão relacionados à necessidade de classificação de risco acurada; administração da unidade de forma organizada; superlotação de atendimentos; sobrecarga de trabalho; déficit no dimensionamento dos recursos humanos, entre outros.

Um estudo de Pereira et al., (2020) realizado com nove profissionais que trabalham na Atenção Pré-Hospitalar (APH), cujo tempo de formação variou entre oito meses a 19 anos, evidenciou que os principais gargalos do serviço que impactam diretamente na humanização da assistência, são: dificuldade de acesso da população com a regulação do SAMU; problemas na estruturação do serviço; falta de capacitação profissional, entreoutros. Em contrapartida, a potencialidade mais evidenciada no estudo foi a autonomia profissional.

A escuta ativa qualificada é uma das principais habilidades cognitivas que o enfermeiro que trabalha na classificação de risco deve contemplar, visando à avaliação do registro correto e minucioso da queixa principal, do pensamento e do raciocínio clínico, além de requerer agilidade mental para a tomadade decisão (Pereira et al., 2020).

\section{Considerações Finais}

As principais fragilidades foram: falta de capacitação profissional, sobrecarga de trabalho, superlotação do serviço e déficit de recursos humanos, falta de equipamentos,má gestão dos leitos credenciados e conflitos interprofissionais. Em relação às potencialidades, observou-se que a escuta ativa e o acolhimento com classificação de risco realizado pelo enfermeiro são 
fatores que contribuem, de forma positiva, para implementação da PNH.

Cabe destacar que o enfermeiro juntamente com sua equipe busca constantemente superar novos desafios. A autonomia está estreitamente relacionada aos saberes próprios da profissão, com a finalidade de executar funções com poder e qualidade, fundamentados no saber científico, reconhecido como o principal instrumento de trabalho do enfermeiro.

Diante do exposto, convém salientar que a classificação de risco é uma importante ferramenta do cuidado em enfermagem, posto que o enfermeiro gerencia a superlotação, pois, é notório que muitos usuários, em função de não receberem um atendimento satisfatório em unidades básicas de saúde ou na Atenção Especializada, acabam recorrendo aos serviços de urgência e emergência. É indiscutível que isso sobrecarrega esses serviços, à medida que, talvez não esteja claro para o usuário o fluxo que ele deve percorrer dentro da rede de atenção à saúde.

Torna-se indispensável a realização de novos estudos que abordem o tema discutido, uma vez que estas práticas se mostram extremamente importantes para a evolução profissional, refletindo em atendimento humanizado a partir de novas atualizações para a implementação ao ambiente de trabalhos dos profissionais e gestores em saúde.

\section{Referências}

Almeida, S. L., Camargo, C., Araújo, K. A., Alves, A. P. B., \& Barreto, H. C. S. (2019). Política de humanização (HumanizaSUS): uma política transversal na saúde. Revista Eletrônica Acervo Saúde, 1(30), e786.

Amestoy, S. C., et al. (2016). Exercício da liderança do enfermeiro em um serviçode urgência e emergência. Revista Eletrônica Gestão e Saúde. 7(1), 38-51.

Andrade, G., et al. (2017). Política Nacional de Humanização. 2(1).

Assis, L. R. S., et al. (2016). Atendimento humanizado no serviço de urgênciae emergência: uma revisão sistemática. Revista Uningá Review. $25(1)$, 131 - 135.

Bittencourt, C. S., \& Ataíde, M. A. (2019). As redes de atenção à saúde em urgência e emergência e sua efetividade nas redes intersetoriais no distrito de Ermelino Matarazzo. Serviço Social e Saúde. 18(esp), e019004-e019004.

Brasil. (2019). Ministério da Saúde/Conselho Nacional de Saúde. RESOLUÇÃO No 617, DE 23 DE AGOSTO DE 2019. 145(1), 52.

Brasil. (2013). Ministério da Saúde. Manual Instrutivo da Rede de Atenção às Urgências e Emergências no Sistema Único de Saúde (SUS). Secretaria de Atenção à Saúde, Departamento de Atenção Especializada.

Ferreira, J. D. O., et al. (2021). Estratégias de humanização da assistência no ambiente hospitalar: revisão integrativa. Revista Ciência Plural. 7(1), 147-163, 2021.

Gerônimo, A. G. S., Monteles, A. O., \& Girão, A. L. A. (2020). Avaliação da implementação dos protocolos de segurança do paciente pela equipe de enfermagem em urgência e emergência. Brazilian Journal of Health Review. 3(4), 10775-10787.

Leite, T. E. H. P., et al. (2018). Os desafios da humanização dentro deunidades de pronto atendimento: a visão dos gestores. Ciência, Cuidado e Saúde. 17(2), $1-8$.

Mendes, T. J. M., et al (2018). Associação entre o acolhimento com classificação de risco, desfecho clínico e o escore Mews. REME rev. min. Enferm. 22(esp), e- 1077 .

Moraes, C. L. K., Neto, J. G., \& Santos, L. G. O. (2020). A classificação de risco em urgência e emergência: os desafios da enfermagem. Global Academic Nursing Journal, 1(2), e17.

Morais, L. A., et al. (2016). Competência legal do enfermeiro na urgência/emergência. Enferm. foco (Brasília), 7(1)., 18-23.

Oliveira, A. D. S., et al. (2018). Avaliação do grau de superlotação de serviçohospitalar de urgência. Revista de Enfermagem da UFPI. 7(2), 41-45.

Pereira, A. B., et al. (2020). Fragilidades e potencialidades laborais: percepção de enfermeiros do serviço móvel de urgência. Revista Brasileira de Enfermagem. 73(5), e20180926.

Pereira, L. C., et al. (2020). Atuação do enfermeiro no serviço de atendimento pré-hospitalar: potencialidades, fragilidades e perspectivas. Research, Society and Development. 9(4), e119942926 - e119942926.

Pereira, M. G. N., Ruzzon, E. D., \& Martins, E. A. P. (2018). O atendimento de urgencia e emergencia por hospital de média complexidade: Revisão integrativa. Revista Internacional de apoyo a la inclusión,logopedia, sociedad y multiculturalidad. 4(2), $42-54$.

Rodrigues, M. C., et al. (2019). Acolhimento com classificação de risco emunidades de pronto atendimento: avaliação sob a ótica do enfermeiro. Saúde em Revista. 19(51), 101-107.

Roncalli, A. A., et al. (2017). Protocolo de Manchester e população usuária na classificação de risco: visão do enfermeiro. Revista Baiana de Enfermagem. 31( 2), 1-10. 
Research, Society and Development, v. 10, n. 13, e346101321536, 2021

(CC BY 4.0) | ISSN 2525-3409 | DOI: http://dx.doi.org/10.33448/rsd-v10i13.21536

Silva, J., \& Vital, S. (2020). Superlotação dos serviços de urgência eemergência hospitalar. Enfermagem Brasil. 19(1), 49-57, 2020.

Silva, A. M. S. M., \& Invenção, A. S. (2018). A atuação do enfermeiro no atendimento de urgência e emergência. UNILUS Ensino e Pesquisa. $15(39), 5-13$.

Silva, J. T., \& Vriesmann, L. C. (2019). Educação Permanente Em Saúde Em Serviços De Urgência E Emergência Hospitalar. Revista Saúde e Desenvolvimento. 13(14), 154-172.

Soares, A. C. L., Brasileiro, M., \& Souza, D. G. (2018). Acolhimento com classificação de risco: atuação do enfermeiro na urgência e emergência. Revista Recien-Revista Científica de Enfermagem. 8(22), 22-33.

Sokolski, B. L., Vandresen, F., \& Senff, C. O. (2019). Desafios da enfermagem para atuação em urgência e emergência. Saúde e meio ambiente: revista interdisciplinar. 8( esp), 207-218.

Souza, C. C. (2017). Atuação do enfermeiro na classificação de risco emserviços de urgência e emergência e a segurança do paciente. Revista de Enfermagem do Centro-Oeste Mineiro, 7(4), 1-2.

Vasconcelos, M. F. F., et al. (2016). Entre políticas (EPS-Educação Permanente em Saúde e PNH-Política Nacional de Humanização): por um modo de formar no/para o Sistema Único de Saúde (SUS). Interface-Comunicação, Saúde,Educação. 20(59, 981-991. 